\title{
Focus on climate change and mental health
}

\author{
The health impacts of climate change are being increasingly recognized, but mental health is often excluded from \\ this discussion. In this issue we feature a collection of articles on climate change and mental health that highlight \\ important directions for future research.
}

oss and trauma feature prominently in media coverage of natural disasters, from forest fires to hurricanes, as affected residents experience personal injury, damage to or loss of personal property, or, in the worst scenarios, the loss of a loved one. As natural disasters increase in frequency and severity due to climate change, it is increasingly important to understand the short- and long-term impacts such experiences may be having on mental well-being, and how these could be mitigated. For instance, experiencing extreme weather events is associated with higher incidence of depression, posttraumatic stress disorder and anxiety, particularly when those who are displaced have little advanced warning ${ }^{1}$.

It is not that climate change is causing new classifications of psychiatric disorders, but rather that it exposes people to circumstances that aggravate known risk factors. For instance, Puerto Rico was already experiencing increases in mental illness due to poverty and family separation prior to Hurricane Maria in September 2017, but destruction from the hurricane further intensified these problems ${ }^{2}$. Climate change can also create situations that disrupt treatment of existing conditions. For example, displacement can interfere with access to medication, and some psychiatric drugs are rendered ineffective by extreme heat or exacerbate heat-related health problems because they interfere with the body's ability to thermoregulate ${ }^{3}$.

However, losses from climate change are not always sudden or associated with specific events. Climate change also exerts more gradual influences on landscapes and ecosystems that may be associated with feelings of loss, particularly for those with close ties to the natural environment. In a Perspective in this issue, Ashlee Cunsolo and Neville Ellis describe research on ecological grief - the grief felt in relation to experienced or anticipated ecological losses. Particularly striking are the similarities between the two groups that Cunsolo and Ellis have studied in this context. It is difficult to think of two environments more different than those inhabited by the Inuit of Northern Canada and farmers

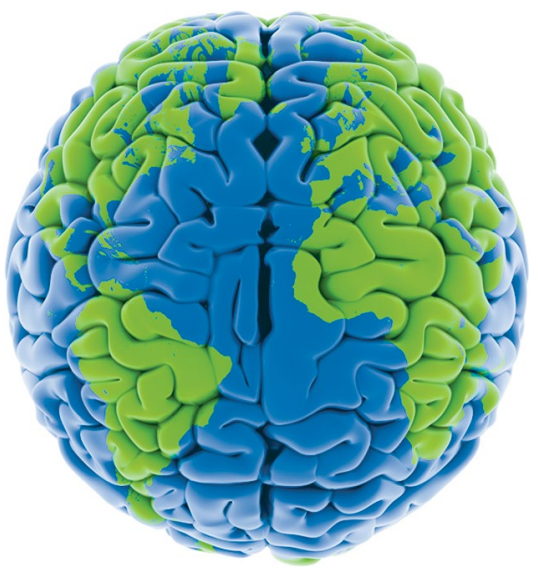

Credit: Henrik5000/E+/Getty

in the Australian Wheatbelt. Yet, experiences of grief from the loss or anticipated future loss of physical ecosystems, environmental knowledge and cultural identity in these two groups in response to different climatological stressors are remarkably similar.

Cunsolo and Ellis highlight the need to understand the personal, lived experience of loss that accompanies climate change. In contrast, in another Perspective in this issue, Helen Berry and colleagues view the issue from the opposite side of the spectrum: they suggest that mental health is an epidemiological problem that requires understanding at the population level within the larger set of causal systems that link mental health risk and climate change exposure. This systems-thinking approach requires integration of direct proximate influences, and indirect interconnected distal influences. For instance, natural disasters exert direct influences on an individual's mental health, as described above, but they also damage critical infrastructure, which diverts resources from public health, puts pressure on individual resources and impairs social cohesion, all of which can ultimately contribute to negative mental health outcomes.

Both individual- and population-level inquiries are important for developing adequate responses to the mental health impacts of climate change. Consider, for instance, a recent paper reporting that warming temperature trends may be responsible for nearly 60,000 suicides in India over the past three decades ${ }^{4}$. Identifying the causal mechanism underlying the relationship between extreme heat and suicide within the complex, context-specific system in which this relationship is embedded is critical for developing sensible policies that protect at-risk populations in the future. But understanding the lived experiences of grief and despair that drive individuals to suicide is needed to provide coping mechanisms for those already suffering.

Rounding out the collection of pieces, Susan Clayton considers whether climate scientists themselves face unique mental health risks because they are immersed in depressing information that threatens something they value and their professional identity. Although this narrative has been popularized in popular media reporting ${ }^{5,6}$, the evidence remains anecdotal, and these articles downplay the more optimistic perspective that Susan Clayton highlights: there are equally good reasons to think that climate scientists benefit from unique protective factors, in particular the social support and experience of collective action that comes from being involved in this research community.

Although the three pieces in this issue explore different ways in which climate change can impact mental health, at different levels, and in different groups, they converge on one key theme: more research is needed.

Published online: 3 April 2018 https://doi.org/10.1038/s41558-018-0128-7

\footnotetext{
References

. Munro, A. et al. Lancet Planet. Health 1, el34-e141 (2017).

2. Dickerson, C. After hurricane, signs of a mental health crisis haunt Puerto Rico. The New York Times (13 November 2017); http://go.nature.com/2oMfbA8

3. Hansen, A., Bi, P., Nitschke, M., Ryan, P., Pisaniello, D. \& Tucker, G. Environ. Health Perspect. 116, 1369-1375 (2008).

4. Carleton, T. Proc. Natl Acad. Sci. USA 114, 8746-8751 (2017).

5. Richardson, J. H. When the end of human civilization is your day job. Esquire (7 July 2015); http://go.nature.com/219Z3jV

6. Yong, E. How coral researchers are coping with the death of reefs. The Atlantic (21 November 2017); http://go.nature.com/2FXtvgo
} 